\title{
Authors' Reply to the Letter to the Editor "Validity of an Alcohol Use Disorder Identification Test: Methodological Issues"
}

\author{
Jung Wei Chang, Jong Sung Kim*, Jin Gyu Jung, Sung Soo Kim, Seok Joon Yoon, Hak Sun Jang \\ Department of Family Medicine, Research Institute for Medical Sciences, Chungnam National University School of Medicine, Daejeon, Korea
}

First and foremost, in our study entitled, "Validity of Alcohol Use Disorder Identification Test-Korean revised version for screening alcohol use disorder according to Diagnostic and Statistical Manual of Mental Disorders, fifth edition criteria," published in the November 2016 edition of the Korean Journal of Family Medicine, we did not conduct any analysis related to the reliability of the Alcohol Use Disorder Identification TestKorean revised version (AUDIT-KR) and did not present any indicator of reliability in the body (Introduction, Methods, Results, and Discussion sections) of our manuscript. Nevertheless, the sentence, "The AUDIT-KR has high reliability and validity for identifying alcohol use disorder according to Diagnostic and Statistical Manual of Mental Disorders, fifth edition (DSM-5) criteria," presented as a concluding statement in the Abstract includes the word 'reliability. We admit that this is a serious clerical error on our part. As mentioned in the Letter, reliability (precision and calibration) is a different methodological issue from validity (accuracy and discrimination). We apologize to the Journal readers for making the mistake of using the word 'reliability' in the Abstract.

As specified in our study, the purpose of the research was to investigate the appropriate cutoff AUDIT-KR scores for screening alcohol use disorder with the newly released DSM- 5 criteria and to evaluate sensitivity, specificity, and positive and negative predictive values. These indicators are included in
'The validity of a single test' section, as mentioned in the Letter. We think it is medically essential to evaluate the necessity of resetting screening standard scores for AUDIT-KR, which has been used as a screening tool for alcohol use disorder based on the DSM- 5 criteria, in accordance with the changed diagnosis criteria. Evaluation of the content or structure validity of the questionnaire itself, as suggested in the Letter, is outside the scope of the purpose of our study.

We could have improved our study if we presented the figures mentioned in the Letter, such as positive and negative likelihood ratios, and odds ratio (ratio of true results to false results). However, we did not do so because of our insufficient statistical knowledge. We totally agree with the opinion expressed in the Letter that inappropriate use of the word 'reliability' may confuse the Journal readers about the concept of validity and reliability. We deeply appreciate the contributor of the Letter to the Editor for pointing out our major mistake and insufficient knowledge on the statistical concept including the use of area under the receiver operating characteristic curve.

\section{CONFLICT OF INTEREST}

No potential conflict of interest relevant to this article was reported. 